\title{
EXTRACTION OF STRUCTURAL ELEMENTS OF INVENTIONS FROM RUSSIAN-LANGUAGE PATENTS
}

\author{
Dmitriy M. Korobkin, Sergey S. Vasiliev, Sergey A. Fomenkov and Vladimir I. Lobeyko \\ Volgograd State Technical University, Russia, Volgograd, Lenin av., 28
}

\begin{abstract}
The paper investigates the issue of extracting structured data from Russian-language patents in the field of new technical solution synthesis. The predicate-argument constructions that characterize the composition of the structural elements of the inventions and the relations between them are the extracted entities. The paper also analyses existing natural language processing tools as applied to patent processing. The authors have offered a new method for extracting predicate-argument constructs taking into account the specificity of the text of patents based on shallow parsing and segmentation of sentences. The value of the F1 metric with a rigorous and lax evaluation of data extraction is $63 \%$ and $79 \%$, respectively, which suggests that the proposed approach is promising. The extracted structures are used to construct the graph of the structural elements of the invention.
\end{abstract}

\section{KEYWORDS}

Patents, Information Extraction, SAO, Claims, CAI-Systems, Shallow Parsing

\section{INTRODUCTION}

In recent years, automation development of inventions has been gaining momentum, and automated support systems are used to search for new technical solutions (Korobkin, 2018a) - CAI systems (Computer-Aided Invention - the search for innovative solutions using a computer). The success of such systems largely depends on the completeness of the ontologies of the subject areas and the fullness of the various knowledge bases that allow generating new technical solutions. However, data enrichment is often a very laborious process. Therefore, an active search continues for effective means of extracting structured data for this purpose. The sources of technical information may be a patent array. However, current approaches to extracting structured data and natural language processing (NLP) tools are poorly oriented to work on an array of patents. In this regard, it is necessary to develop new efficient methods for extracting data from patents.

The task of information support for new technical solution synthesis (Korobkin, 2018b) can be seen as the task of extracting the SAO (Subject - Action - Object) semantic structures. In particular, elements of the design of a technical object (TO) and the relationship between them are considered as the source of information support. Claims are considered to be a direct source of data for retrieval. They express the essence of the invention and are based on a description; therefore it is of the greatest interest for the extraction of technical information. In this case, the object of analysis is an array of Russian-language patents.

\section{RESEARCH BACKGROUND}

Various systems (Choi, 2011, Guo, 2016, Wang, 2015, Yang, 2017) are known for processing English-language patents, including the use of the SAO formalism to extract various concepts. In (Yang, 2017), a tree syntax analysis was applied with a separate identification of the subject, the action and the object to improve the quality of parsing. The authors state the following averaged values of precision and recall: 0.8058 and 0.8446 , respectively. At the same time, the project was implemented in the GATE system, 
which is poorly suitable for the Russian language. In (Wang, 2015), the SAO structures are extracted based on the rules using the Stanford parser software, but there are no ready-made models for the Russian language. Papers (Guo, 2016, Souili, 2015) process patents using linguistic markers (specific verbs and nouns) and lexical-syntactic patterns, while (Souili, 2015) notes the need to study the structure of patent documents to improve the quality of data extraction. The emphasis is mainly on rule-based systems, since for statistical analysis systems a lot of marked-up data is obviously needed. This causes additional development difficulties.

The specific style of writing a patent claims complicates the work of existing parsers. Highlight the problems: (a) the length of the sentences of the claims is excessively long; (b) the claims are described predominantly with noun-phrases, often with a chain of definitions and participial constructions; (c) the availability of specific words and constructions can affect the correct operation of NLP tools.

Considering the system for extracting structured data from Russian-language texts, the development of Yandex Tomita-parser is most often distinguished. Extraction takes place according to the rules, written in an extended context-free grammar. Despite the expressive power of context-free grammars and the Tomita-parser tool, the question of organizing the effective extraction of SAO structures from the claims remains open.

Of the available systems of syntactic and semantic-syntactic analysis, for which there is an opportunity to work with the Russian language, we can distinguish Link Grammar Parser, MaltParser and UDPipe (UFAL, 2019).

Among the available Russian language analyzers can be identified: TreeTagger, MyStem, TnT, pymorphy2, FreeLing. A number of works (Asiryan, 2017, Blazhievskaya, 2016, Dereza, 2016) are devoted to the comparison of morphological analyzers of the Russian language. In the works (Blazhievskaya, 2016, Dereza, 2016) on the quality of determining the parts of speech (POS), TreeTagger is best distinguished, but they do not take into account the work of the Yandex MyStem tool. In (Asiryan, 2917), already taking into account MyStem, the latter was chosen as the best tool with the F1 metric value of $94 \%$. At the same time, an experiment is being conducted to improve the quality of parsing by combining tools (correcting MyStem with pymorphy 2 with an increase in F1 to 95\%). However, the full use of the analyzer MyStem is difficult due to the issuance of grammatical signs in the form of an unordered list of alternatives. At the same time, there are no attributes for the correct choice of a single grammatical form, the alternatives simply follow in alphabetical order. The TreeTagger morphoanalyzer is devoid of such a feature - the grammar is issued without alternatives. The disadvantage of TreeTagger is that its speed is somewhat lower than the speed of processing MyStem on large texts.

Having considered certain aspects of the processing of Russian-language texts, we summarize the problems that arise when extracting structured data (SAO structures) from texts of Russian-language patents when solving the problem of information support for automated invention: (a) the claims are written in a specific sublanguage of patents that impede text analysis (specific terminology); (b) the structure of the claims is poorly suited for parsing by ready-made syntactic analyzers (given the prevalence of sentences and the presence of multiple participle clauses); (c) the structure of the claims is not fully decomposed by simple heuristics; (d) the available NLP tools will certainly be mistaken in the analysis, so relying on one tool in the processing of complex structures is not always rational.

The presented limitations make it difficult to create an efficient data extraction system from patent claims using publicly available NLP tools (without considering complex commercial or closed systems).

However, given that the texts of patents are written in a template (not only in the structure of sections, but also in the structure of phrases), and it is possible to combine NLP tools for mutual correction of analysis errors; the authors suggest the following approach to processing the text of the claims, including the steps:

- segmentation of the patent claims based on shallow parsing;

- organization of the extraction of SAO structures based on the valence bond theory;

- post-processing of SAO structures with linking concepts to a common repository.

\section{APPROACH DESCRIPTION}

As the source of data for the extraction of technical descriptions, the main clause of the multi-tier claim of the invention on the device in the patent text is selected. The unit of extraction is the predicate-argument 
construction in the form of the SAO structure, which semantically describes the structural elements of the device and the connections between them.

As instruments of NLP in the experimental mode are used: (a) UDPipe (tokenization, tagging, lemmatization and syntactic analysis); (b) MyStem (lemmatization, correction of morphology); (c) pymorphy2 (Korobov, 2015) (declination of word forms); (d) Chanker of noun phrases of Russian (to define the boundaries of actants).

The ultimate goal of extracting data is to build a related graph of structural elements of the invention, reflecting the structure of the invention itself. Let us consider in more detail each of the stages.

\subsection{Segmentation of Patent Claims}

On the basis of a well-established patents language, the authors made the assumption that it is possible to identify certain patterns of segments in a patent claims, and that shallow parsing with a number of heuristics will be enough to successfully extract the necessary structures.

The segment of the independent claim will be called the element of the sentence, which is indicated by punctuation (comma, and/or semicolon). In the Russian language, such segments can act as simple-main sentences, be subordinate clauses, participal and verbal adverb phrases, isolated definitions, etc. In addition, one should take into account the logical division of the claim into the restrictive and distinctive parts (if such separation exists), usually demarcated by the construction of "characterized in that".

The purpose of segmentation is to identify the linear structure of the proposal of the claims for the subsequent extraction of SAO-objects based on the analysis of the morphological and linear-combinatorial characteristics of the text. Let us explain it with the example of the text of the patent "Pressure sensor", the syntactic analysis of which is presented in Figure 1.

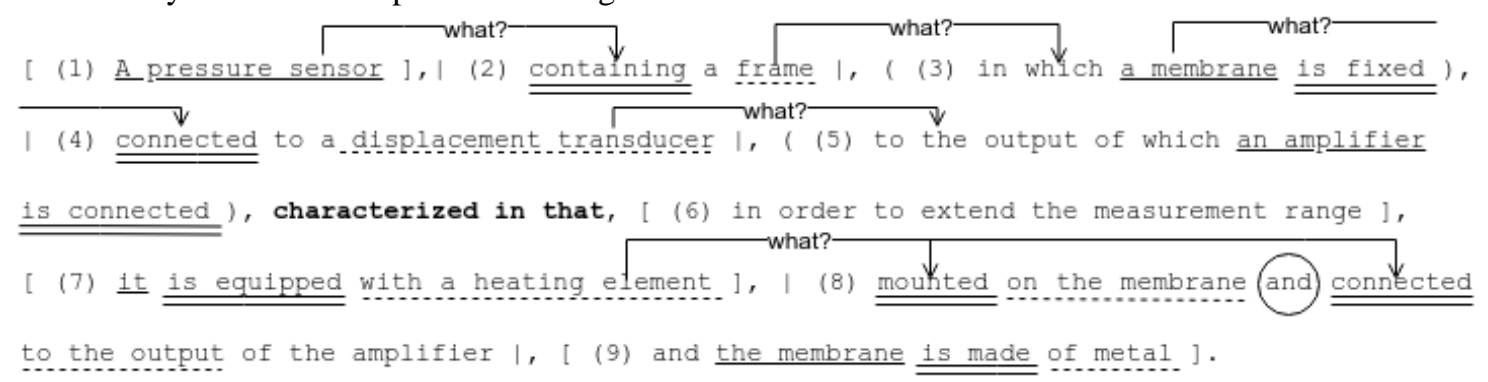

Figure 1. Parsing sentence

In parentheses are marked linear numbers of segments without taking into account the demarcation design "characterized in that". At the same time, the verb forms without specifying the role in the sentence are highlighted with a double line.

The sentence includes: generic term (1); participle clause $(2,4,8)$; subordinate-attributive $(3,5)$; simple two-part $(7,9)$. The tasks of segmentation include the identification of segments by composition and the definition of the relationship between the main and dependent words. In the future, this information is necessary for the direct extraction of SAO-objects.

To identify the nature of writing segments and formalize the definition of their types, a set of fourteen main claims was taken, mainly from different sections of the international patent classification (IPC). The total number of segments in the sample was 187 elements.

After a detailed manual analysis, the following conditional segment types were derived (see Table 1).

At the same time, the symbol for the segment was formed from acronyms, taking into account the basic structure of the segment, i.e., the POS and / or elements that characterize it: Noun (N); Verb (V); Adjective (A); "which" is a demonstrative pronoun in subordinate clauses; Adverb (AV); and others. The legend of the segments in the future act as markers in the system.

Apparently from results of the analysis, all the segments met a finite number of fairly typical sentence constructions. Of course, it is not possible to thoroughly analyze the entire array of patents manually. Therefore, the authors made the assumption that the number of types of segments (in terms of the overall structure of writing) of a larger mass of the claims is also limited. 
Table 1. Types of segments of the test sample claims

\begin{tabular}{lll}
\hline \multicolumn{1}{c}{ Type of segment } & \multicolumn{1}{c}{ Symbol } & \multicolumn{1}{c}{ Example of a segment } \\
\hline Noun phrase & N & 'contact electric current modulator' \\
Participle clause & V_N & 'containing vibration transducer' \\
Independent segment & N_V_N & 'it is equipped with an additional vibration converter' \\
Isolated attribute & A0 & 'identical to the main' \\
Rather independent segment & (N_) which_V_N & 'which input is the input of the device' \\
Dash instead of a predicate & N_-_N & 'and the cathode through resistance - with the anode' \\
Comparative turns & AV & 'less' \\
Subordinate clauses & SC & 'that they have sufficient fatigue strength' \\
Verbal adverb phrase & V<C> & 'preventing the deformation of the first destructive elements' \\
Service segments & SEPARATOR & besides; and; and also; at the same time; \\
& NAMED & 'called decaying elements' \\
& PURPOSE & 'for the purpose of range extension of measurements' \\
\hline
\end{tabular}

Since the segmentation is carried out in the context of the task of extracting predicate-argument structures, the elements of this extraction are partially embedded in the process in the form of highlighting non-significant (service) segments and the target semantic classes of verbs.

Semantic classes of verbs are needed to find to SAO target ligaments, potentially describing constructive elements and the connections between them. A samples of such verbs is presented in Table 2 .

Table 2. Semantic classes of verbs

\begin{tabular}{ll}
\hline \multicolumn{1}{c}{ Semantic class } & \multicolumn{1}{c}{ Verbs } \\
\hline Existence of a structural element & $\begin{array}{l}\text { contain (on); equip; include (between; in; with; in;); have; supply; enter (into); } \\
\text { consist (from); } \\
\text { Inter-element couplings }\end{array}$ \\
set (on; as); connect (to); fasten (in; on); place (in; on);
\end{tabular}

The overall sequence of operations for the segmentation of the claims:

1) Text pre-processing.

2) The process of obtaining initial segments is associated with sequential fragmentation of the text by punctuation marks with auxiliary treatments. A specific feature of the stage is the final fragmentation based on the results of the syntactic analysis obtained after using the UDPipe tool. Tokenization is also performed by this tool. This decision is justified by the need to take into account the context for better analysis: submitting partially fragmented text to the analyzer input (based on strong signs of separation - for example, different parts of the claims or a semicolon), but without carrying out fragmentation on commas, it is possible to receive more adequate syntactic marking; analysis of segments completely isolated from each other potentially also leads to errors.

3) The next step is the process of correcting morphology. If a segment token refers to parts of a verb, adjective, or adverb speech, then a part of speech is checked using MyStem. Moreover, if the result does not coincide with the initial one, then the morphological information of the token is updated taking into account the conversion of tags from MyStem into UDPipe format (CoNLL-U).

4) One of the fundamental stages of segmentation is the determination of the type of segment (see Table 1). The type of segment allows you to introduce certainty in the use of certain heuristics processing in the future. This problem is solved in two steps: the determining of a segment pattern and the determining the type of segment by pattern using a finite state machine (FSM).

The definition of a segment pattern is based on finding out its main composition; what is based on the priority of the presence of POS. Example of the dictionary of symbols in the template is presented in Table 3.

Table 3. Template dictionary

\begin{tabular}{|c|c|c|}
\hline Symbol & Part of speech & Note \\
\hline $\mathrm{N}$ & noun & Index 0 means accusative or a nominative case \\
\hline $\mathrm{V}$ & verb & $\begin{array}{l}\text { Possible litters: }\langle\mathrm{P}\rangle \text { - participle; }\langle\mathrm{F}\rangle \text { - finite verb; }\langle\mathrm{C}\rangle \text { - adverbial participle; }\langle\mathrm{I}\rangle \text { - } \\
\text { infinitive; }\langle\mathrm{U}\rangle \text { - undefined. }\end{array}$ \\
\hline A & adjective & Index 0 means the top of the sentence (indirect attribute) \\
\hline PR & pronoun & $\begin{array}{l}\text { Index } 0 \text { means the nominative case } \\
\text { Direct replacement of the word «which» is possible }\end{array}$ \\
\hline
\end{tabular}


5) Binding of segments. The main binding mechanism in the Russian language is coordination according to gender, number, and case. Therefore, the segmentation is carried out on the data of the extracted morphology, and the task is reduced to finding this agreement between the words in the sentence. At the same time, segment types limit the set of binding rules.

Proceeding from a linear logic of the description of the formula, it is possible to connection of segments in one straight pass (from left to right). An exception is the case of a rupture of the sentence by an enclosed turnover. As a rule, the second part of a broken sentence begins with finite verb. When finding such a segment, it is necessary to find the closest missing segment of the noun group to the left. The assembly of such segments precedes the main straight passage.

The result of segment linking for an example (Figure 1) is shown in Figure 2.

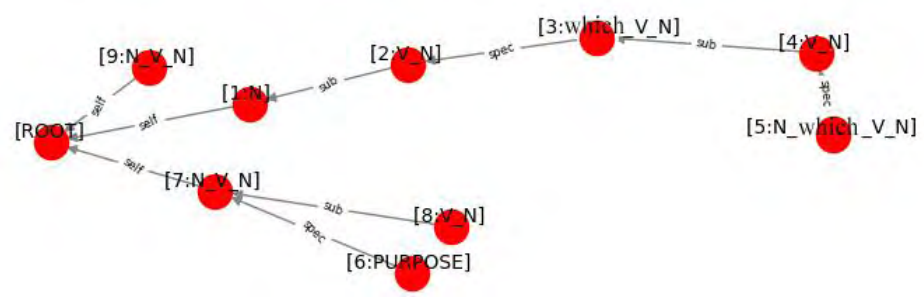

Figure 2. The graph of segments for an example

\subsection{Extracting SAO Primary Structures}

The extraction of predicate-argument constructions is based on the results of segmentation and valency of the target verbs (see Table 2). The valence of the verb determines the number of possible arguments. It is enough to define the "subject" and "object" of the structure being extracted.

The subject can be determined by the following cases (see Example 1): by the nominative case (1), by the word of the participle being defined (2) or it can be completely absent (3), implying a generic term.

Example 1. The definition of the subject in the segments.

(1) The magnetic gearbox is equipped with a rotor ...

(2) ... magnetic gearbox, equipped with a rotor ...

(3) ... characterized in that ..., a rotor is introduced, ...

The definition of the object is somewhat complicated due to the lack of explicit universal expression in the predicate arguments. The role of an object in a predicate corresponds to a specific case, which can be formed using prepositions.

Consider the cases of determining the object from Example 2: accusative case without preposition (1), dative case formed with the preposition "to" (2), several actants with prepositions (3); in the latter case, the preposition "through" generates an adverb (connected as? - "through a non-magnetic ring"), the target actant is a combination with the preposition "with" (connected with what? - "with the body").

Example 2. The definition of the object in the structure,

(1) including shaper

(2) connected respectively to the output of the amplifier

(3) connected with the body through a non-magnetic ring

It should also be taken into account that the predicate can be expressed by a verb with a compound preposition (for example, "made in the form of"). In addition, information on binding to the predicate can already be partially determined at the end of the segmentation.

When detected in the segment of the target verbs (see Table 2), the sequence of currents to the right and left of the verb extends to the left and right parts, respectively. For full-length segments (types "N_V_N", "N_which_V_N", "which_V_N" and "N__- N" from Table 1), the right and left parts are formed in the same segments, and for subordinates (type "V_N") the left part is replaced and forms the found subject connecting.

Next comes the key stage of extraction: the search for the structures of the object and subject in the selected parts of the segment according to the predicate valencies. The order of searching for a subject and an object is determined by the possible position of actants indicated in valency (the "entry" field).

The search for valencies in the definition of a subject and an object in parts of a segment is carried out using the token-marking mechanism. The following label sets are used: 
- for the subject (P-SBJ, N-SBJ, I-SBJ and A-SBJ);

- for an object (P-OBJ, N-OBJ and I-OBJ)

- for auxiliary valencies (P-ON, P-VIA, N-ON, N-VIA, N-BY, N-WHOM).

The label prefix corresponds to the functional purpose of the token: P- (Preposition); N- (Noun), A- (Adjective) - the top of the noun group; I- (Inside) - the entry of a token into a noun group.

Marks in parts of the segment are placed for the object according to the predicate valences, and for the subject - for the nominal case of a noun (or less often an adjective).

If there is a mandatory preposition in valency, the occurrences of the specified preposition are first sought, in case of failure, the search is interrupted. The search for additional valences for an object is more priority than the search for the valence of the object itself, i.e. starts first.

\subsection{Creation a Tree of Relations of Elements of Structures}

Creation a tree of relationships requires determining the final vertices of the graph, taking into account the presence of identical concepts, as well as identifying implicit relations of generic terms between elements.

For further binding of the primary SAO objects, preprocessing is necessary:

- the separation of homogeneous members in the description of structural elements along the border of the composing union "and" provided it is located between the labels of the vertices of the noun group (ie, "N-SBJ" or "N-OBJ").

- generation of the normal form of the actants (in the nominative case) and the genitive case (further as the Gent-form).

- after preprocessing, the primary SAO object is considered prepared.

Actants are declined using the pymorphy 2 tool. This is followed by linking the prepared SAO objects into a single graph. The mechanism consists in the sequential transformation of actants of subjects and objects into a set of anchor points from a common glossary of terms, followed by memorization of the relation (predicate) for the identified points of the subject and object.

The general graph of relations is stored in the form of a dictionary of terms, a dictionary of relations and a map of relations containing the parental relations between the actants through predicates (from the dictionary of relations).

Anchor points are formed when analyzing the subject and object of the prepared SAO objects. When terms are added (the normal form of an actant's sentence), the parent relationship with another term is searched. The operation is carried out under the condition that the normal form of the actant has a sequence of tokens in the genitive case (marked with an "I-GEN" label in the analysis). An example of a parent relationship search is shown schematically in Figure 3.

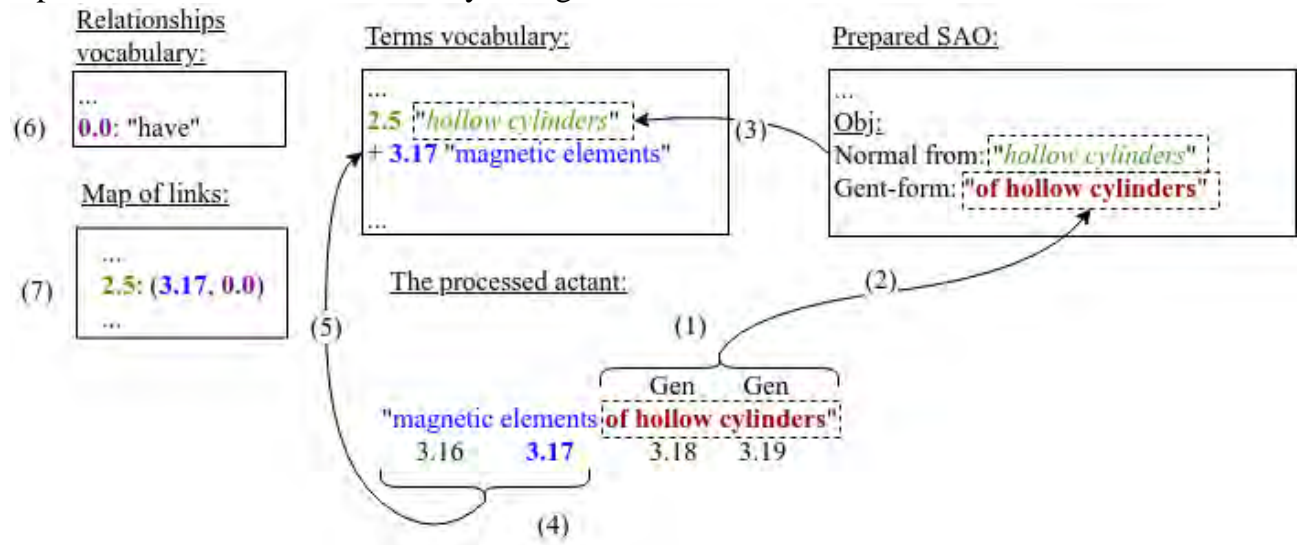

Figure 3. The search logic of the parent relationship

If the final sequence of tokens in the processed actant is in the genitive case (1), then the word forms in the array of prepared SAO objects (2) are checked for coincidence. The parent actant index in the terms vocabulary (3) is restored by the bundle identifier or normal form. The processed actant is cleared from the gent-ending and a new actant is added to the terms vocabulary (5). The "have" parent relationship is already 
included by default in the relationships vocabulary (6). Next, the map of links is updated to reflect the new ratio of anchor points (7).

In this case, the indices in the vocabularies are the coordinates of the vertex of the noun group (including the segment number and the token number in the segment).

After receiving the anchor points, consisting of the coordinates and the normal form of the structural elements, the map of connections is filled: the actants of the subject act as parent concepts for the actants of the object connected through the predicate. The results of processing the prepared SAO objects are buffered, and after checking the semantic classes of actants, the graphs of the entire device are updated.

\section{SOFTWARE EVALUATION METHODOLOGY}

The quality of data extraction was assessed for primary SAO objects (i.e., excluding post-processing with the separation of homogeneous members and taking into account the semantic classes of actants). For this purpose, a manual marking of an independent test set was carried out, including the main points of the claims 30 patents. The markup was made taking into account the semantic classes of verbs introduced into the system, which characterize the target relations between the components of the technical object. In this case, it was allowed to add into the system descriptions (valencies) of previously not encountered target verbs.

The following metrics were used as evaluation metrics: precision, recall and F1-measure. A test data set of 30 documents on the basis of manual marking included 318 SAO objects. The results of counting the number of extracted test data set objects are presented in Table 4 (first row). The design data set of 14 documents contained 136 recoverable structures. The results of counting the number of extracted SAO objects of the design data set are presented in Table 4 (second row).

Table 4. Extracted test data set objects

\begin{tabular}{ccccc}
\hline Parameter & $\begin{array}{c}\text { Rf (all SAO } \\
\text { structures } \\
\text { extracted by the } \\
\text { system) }\end{array}$ & $\begin{array}{c}\text { Rrel_1 (relevant SAO } \\
\text { structures with a rigorous } \\
\text { entry of the entire noun } \\
\text { group in the argument }\end{array}$ & $\begin{array}{c}\text { Rrel_2 (relevant SAO } \\
\text { structures taking into account } \\
\text { that the vertex of the noun } \\
\text { group are in the argument) }\end{array}$ & $\begin{array}{c}\text { Srel (manually selected } \\
\text { SAO structures) }\end{array}$ \\
\hline Amount & 305 & 198 & 248 & 318 \\
Amount & 142 & 118 & 129 & 136 \\
\hline
\end{tabular}

The result of the counting of the extraction quality metrics (according to formulas $1-3$ ) for the test and design data set is presented in Table 6.

Table 6. Metric counting results

\begin{tabular}{lcccc}
\hline \multirow{2}{*}{ Metrics } & \multicolumn{2}{c}{ Test data set } & \multicolumn{2}{c}{ Design data set } \\
\cline { 2 - 5 } & Lax evaluation & Rigorous evaluation & Lax evaluation & Rigorous evaluation \\
\hline Precision & 0,81 & 0,65 & 0,91 & 0,83 \\
Recall & 0,78 & 0,62 & 0,95 & 0,87 \\
F1-measure & 0,79 & 0,63 & 0,93 & 0,85 \\
\hline
\end{tabular}

\section{CONCLUSION}

Extracting structured data from patents depends on the specifics of the writing of documents. The peculiarities of writing claims of the invention include excessive length of sentences, complication of the participle clauses, characteristic terminology. Practical experience has shown that NLP tools do not always produce a stable result on the domain of patents. Thus, they are ineffective in building high-quality data extraction systems with direct application. Therefore, special approach to the pre-processing of patent texts seems necessary. It is worth noting that in practice they often use a combination of tools to improve the quality of processing. 
Due to the sublanguage of patents and the availability of common templates for the formation of documents, the author made an assumption about the sufficiency of morphological and shallow parsing analysis with a number of heuristics to effectively extract structural elements from the description of the claims.

The developed method of extracting SAO structures involves two main stages: segmentation of the sentences of the claims and the extraction of technical implementations in the form of predicate-argument structures. Segmentation is based on the derivation of typical structures in the sentence and coordination of dependent words on cases. The extraction of structural elements of technical objects is based on a verbs set corresponding to certain semantic classes. After that, the extracted structural elements are linked into a graph, taking into account homogeneous members and parental relations.

\section{ACKNOWLEDGEMENT}

The reported study was funded by RFBR (research project 18-07-01086), RFBR and Administration of the Volgograd region (research projects 19-47-340007, 19-41-340016).

\section{REFERENCES}

Asiryan, A.K., 2017. Morphological tagging tools comparison. Paper presented at the INTELLECTUAL POTENTIAL OF THE XXI CENTURY '2017, November, <https://www.sworld.com.ua/konferu7-317/27.pdf>.

Blazhievskaya, A. et al, 2016. Morphological Analysis for Russian: Integration and Comparison of Taggers. Analysis of Images, Social Networks and Texts. Springer, Vol. 661, pp. 162-171.

Choi, S. et al, 2011. SAO network analysis of patents for technology trends identification: A case study of polymer electrolyte membrane technology in proton exchange membrane fuel cells. Scientometrics, pp. 863-883. doi: $10.1007 / \mathrm{s} 11192-011-0420-\mathrm{z}$

Dereza, O.V. et al, 2016. Automatic Morphological Analysis for Russian: a Comparative Study. Computational Linguistics and Intellectual Technologies: Proceedings of the International Conference «Dialogue».

Guo, J. et al, 2016. Subject-action-object-based morphology analysis for determining the direction of technological change. Technological Forecasting and Social Change, No. 105, pp. 27-40.

Korobkin D., Fomenkov, S., Golovanchikov, A., 2018. Method of identification of patent trends based on descriptions of technical functions Journal of Physics: Conference Series. - 2018. - Vol. 1015. - 7 p.

Korobkin, D., Fomenkov, S., Kolesnikov, S., 2018. The method for detecting the dependencies between technical functions and physical effects Proceedings of theMCCSIS 2018. - [Madrid], 2018. - pp. 225-228.

Korobov, M., 2015. Morphological Analyzer and Generator for Russian and Ukrainian Languages. Analysis of Images, Social Networks and Texts, pp. 320-332, arXiv:1503.07283v1

Souili, A. et al, 2015. Starting from Patents to Find Inputs to the Problem Graph Model of IDM-TRIZ. Procedia Engineering, Vol. 131, pp. 150-161. doi: 10.1016/j.proeng.2015.12.365

UFAL UDPipe, 2019, <http://ufal.mff.cuni.cz/udpipe>.

Wang, X. et al, 2015. Identification of technology development trends based on subject-action-object analysis: The case of dye-sensitized solar cells. Technological Forecasting and Social Change. No. 98, pp. 24-46.

Yang, C. et al, 2017. SAO Semantic Information Identification for Text Mining. International Journal of Computational Intelligence Systems, Vol. 10., pp. 593-604. doi: 10.2991/ijcis.2017.10.1.40 